\title{
Model predictive control for stochastic max-min-plus-scaling systems - An approximation approach*
}

\author{
S.S. Farahani, T. van den Boom, and B. De Schutter \\ If you want to cite this report, please use the following reference instead: \\ S.S. Farahani, T. van den Boom, and B. De Schutter, "Model predictive control for \\ stochastic max-min-plus-scaling systems - An approximation approach," Proceed- \\ ings of the 2011 50th IEEE Conference on Decision and Control and European Con- \\ trol Conference (CDC-ECC), Orlando, Florida, pp. 391-396, Dec. 2011.
}

Delft Center for Systems and Control

Delft University of Technology

Mekelweg 2, 2628 CD Delft

The Netherlands

phone: +31-15-278.24.73 (secretary)

URL: https: / / www.dcsc.tudelft.nl

*This report can also be downloaded via https://pub. deschutter.info/abs/11_040.html 


\title{
Model Predictive Control for Stochastic Max-Min-Plus-Scaling Systems - An Approximation Approach
}

\author{
Samira S. Farahani, Ton van den Boom, and Bart De Schutter
}

\begin{abstract}
A large class of discrete-event and hybrid systems can be described by a max-min-plus-scaling (MMPS) model, i.e., a model in which the main operations are maximization, minimization, addition, and scalar multiplication. Further, Model Predictive Control (MPC), which is one of the most widely used advanced control design methods in the process industry due to its ability to handle constraints on both inputs and outputs, has already been extended to both deterministic and stochastic MMPS systems. However, in order to compute an MPC controller for a general MMPS system, a nonlinear, nonconvex optimization problem has to be solved. In addition, for stochastic MMPS systems, the problem is computationally highly complex since the cost function is defined as the expected value of an MMPS function and its evaluation leads to a complex numerical integration. The aim of this paper is to decrease this computational complexity by applying an approximation method that is based on the raw moments of a random variable, to a stochastic MMPS system with a Gaussian noise. In this way, the problem can be transformed into a sequence of convex optimization problems, providing that linear or convex MPC input constraints are considered.
\end{abstract}

\section{INTRODUCTION}

Conventional control design techniques that generate optimal controllers or control input sequences for the entire future evolution of the system, are often unable to work efficiently once additional constraints on inputs and outputs are included. On the contrary, model predictive control (MPC) is capable of handling constraints on both inputs and outputs in a systematic way while having other important properties such as being an easy-to-tune method, being able to track pre-scheduled reference signals, and being applicable to multivariable systems. MPC uses linear or nonlinear discretetime models [1] as well as discrete-event models [2].

In this paper we consider stochastic max-min-plus-scaling (MMPS) systems in which the main operations are maximization, minimization, addition, and scalar multiplication. A large class of discrete-event and hybrid systems can be described by an MMPS model. Hybrid systems contain both analog (continuous) and logical (discrete, switching) dynamics. Typical examples are manufacturing systems, telecommunication and computer networks, traffic control systems, digital circuits, and logistic systems. Discrete-event systems are systems, the behavior of which is governed by events rather than by ticks of a clock. In [3] it is shown that the class of MMPS systems encompasses several other classes of discrete-event systems such as max-plus linear

The authors are with Delft Center for Systems and Control, Delft University of Technology, Mekelweg 2, 2628 CD Delft, The Netherlands

s.safaeifarahanietudelft.nl;

a.j.j.vandenboometudelft.nl;

b. deschutteratudelft.nl systems. Furthermore, it has been shown in [4]-[6] that MMPS systems are equivalent to a particular class of hybrid systems, namely continuous piecewise affine (PWA) systems. The relation between PWA and MMPS systems is useful for the investigation of structural properties of PWA systems such as observability and controllability but also in designing controller schemes like model predictive control (MPC) [7], [8]. In stochastic hybrid or discrete-event systems, processing times and/or transportation times are assumed to be stochastic quantities, since in practice such stochastic fluctuations can, e.g., be caused by machine failure or depreciation [9]. Some results for the analysis of some specific subclasses of stochastic discrete-event or hybrid systems can be found in [3], [10]-[17], and for stochastic MPC, can be found in [18], [19].

In the last few decades, MPC has been attracted lots of attention and has been applied to MMPS systems. Some related work on controller design, such as MPC, for MMPS systems can be found in [3], [20]. Following the MPC methodology, the optimal input sequence has to be computed in order to minimize the cost function. For a stochastic MMPS systems, in particular, the cost function can be defined as the expected value of an MMPS function. Since there are no closed-form analytic expressions for such an expected value, the computation of the cost function in principle involves numerical integration, which is computationally complex and very time consuming. To the authors' best knowledge no effort has been made to decrease this computational complexity for MMPS systems.

The aim of this paper is to tackle the stochastic MPC-MMPS optimization problem using an approximation method in order to increase the computational efficiency and to decrease the computation time. Using the equivalence between MMPS and PWA systems and the results of [21] and [22] we can rewrite an MMPS function as a difference of two convex functions. Next we can apply the approximation method presented in [23] in order to significantly simplify the computation. In fact, this approximation method is applied to approximate the calculation of stochastic integrals and it is based on raw moments of random variables. By choosing the appropriate order of raw moments, the error of approximation can be made sufficiently small and since we can compute these moments analytically, the computation burden decreases considerably. Accordingly, the problem, which is by nature non-convex, will be transformed into a series of convex optimization problems and can be solved using efficient convex optimization algorithms.

The structure of this paper is as follows. In Section 2 we 
present some background information on stochastic MMPS systems. Section 3 provides a brief description of the MPC algorithm for stochastic MMPS systems. In Section 4 we study concisely the equivalence of MMPS and PWA systems and we express an MMPS function as a difference of two convex functions. Section 5 describes the approximation method based on raw moments and Jensen's inequality, and explores how it decreases the complexity of stochastic MPCMMPS optimization problem. In Section 6 we show in a worked example how the stochastic MPC-MMPS optimization problem can be solved by using the methods proposed in Sections 4 and 5. Section 7 concludes the paper.

\section{STOCHASTIC MAX-MIN-PLUS-SCALING SYSTEMS}

A scalar-valued MMPS function $g: \mathbb{R}^{n} \rightarrow \mathbb{R}$ of the variables $x_{1}, \ldots, x_{n}$ is defined by the recursive relation

$$
\begin{gathered}
g\left(x_{i}\right)=x_{i}|\alpha| \max \left(g_{k}(x), g_{l}(x)\right)\left|\min \left(g_{k}(x), g_{l}(x)\right)\right| \\
g_{k}(x)+g_{l}(x) \mid \beta g_{k}(x),
\end{gathered}
$$

where | stands for "or", $\alpha, \beta \in \mathbb{R}, i=1, \ldots, n$, and $g_{k}$ and $g_{l}$ are MMPS functions. Note that for a vector-valued MMPS function $g: \mathbb{R}^{n} \rightarrow \mathbb{R}^{m}$ each component of $g$ is an MMPS function of the above form.

Accordingly, a state space representation of a deterministic MMPS system can be described as follows:

$$
\begin{aligned}
& x(k)=\mathscr{M}_{x}(x(k-1), u(k)) \\
& y(k)=\mathscr{M}_{y}(x(k), u(k)),
\end{aligned}
$$

where $\mathscr{M}_{x}, \mathscr{M}_{y}$ are MMPS functions, $x(k)$ is the system state at time or event step $k, u(k)$ is the system input, and $y(k)$ is the system output. However, in this paper we study stochastic MMPS systems in which noise and modeling errors are present. Similar to conventional linear systems, disturbances and modeling mismatches appear in the systems equations. The system (1)-(2) then turns into:

$$
\begin{aligned}
& x(k)=\mathscr{M}_{x}(x(k-1), u(k), e(k)) \\
& y(k)=\mathscr{M}_{y}(x(k), u(k), e(k)) .
\end{aligned}
$$

Here, we consider both noise and modeling errors in a single framework and present them by the vector $e(k)$ that is a stochastic variable with a certain probability density function $f$.

\section{MPC FOR MMPS SYSTEMS}

This section gives a brief description of MPC for MMPS systems of the form (3)-(4) (see [3]). Note that in [3] the error vector was assumed to be in a bounded polyhedral set and a worst-case scenario, i.e., the maximal possible objective function, was considered. However, in this paper we assume that the error vector $e(k)$ is a stochastic variable with a Gaussian probability distribution. As a result, we can study stochastic MMPS systems without restricting ourselves to the worst-case scenario. Following the conventional MPC methodology [1], we define a cost criterion $J$ that reflects the reference tracking error $\left(J_{\text {out }}\right)$ and the control effort $\left(J_{\text {in }}\right)$ from step $k$ to $k+N_{\mathrm{p}}-1$ :

$$
J(k)=J_{\text {out }}(k)+\lambda J_{\text {in }}(k)
$$

where $N_{\mathrm{p}}$ is the prediction horizon and $\lambda>0$ is a weighting factor. The aim is to compute the optimal input sequence $u(k), \ldots, u\left(k+N_{\mathrm{p}}-1\right)$ that minimizes $J(k)$ subject to linear or convex constraints on the input (as explained in [3], [20]). Typical choices of $J_{\text {out }}$ and $J_{\text {in }}$ are:

$$
\begin{aligned}
J_{\text {out }, 1}(k) & =\|\tilde{y}(k)-\tilde{r}(k)\|_{1}, \quad J_{\text {out }, \infty}(k)=\|\tilde{y}(k)-\tilde{r}(k)\|_{\infty}, \\
J_{\text {out }, \mathrm{t}}(k) & =\max \{\tilde{y}(k)-\tilde{r}(k), 0\} \\
J_{\text {in }, 1}(k) & =\|\tilde{u}(k)\|_{1}, \quad J_{\text {in }, \infty}(k)=\|\tilde{u}(k)\|_{\infty}, \\
J_{\text {in }, \mathrm{t}}(k) & =-\sum_{i} \tilde{u}_{i}(k)
\end{aligned}
$$

where $\tilde{u}(k)=\left[u^{T}(k), \cdots, u^{T}\left(k+N_{\mathrm{p}}-1\right)\right]^{T}, \tilde{r}(k)=$ $\left[r^{T}(k), \cdots, r^{T}\left(k+N_{\mathrm{p}}-1\right)\right]^{T}, \tilde{y}(k)=\left[y^{T}(k), \cdots, y^{T}(k+\right.$ $\left.\left.N_{\mathrm{p}}-1\right)\right]^{T}$, and $\tilde{r}(k)$ is the reference signal to be tracked. We assume that the state $x(k-1)$ can be measured or predicted at each step $k$ and by successive substitution on (3)-(4) we obtain the system output as

$y(k+j)=\mathscr{M}_{j}(x(k-1), u(k), \ldots, u(k+j), e(k), \ldots, e(k+j))$

for $j=0, \ldots, N_{p}-1$. Clearly $y(k+j)$ is an MMPS function of $x(k-1), u(k), \ldots, u(k+j), e(k), \ldots, e(k+j)$. Define $\tilde{e}(k)=$ $\left[e^{T}(k), \cdots, e^{T}\left(k+N_{\mathrm{p}}-1\right)\right]^{T}$; hence, from (6) we conclude that all criterion functions in (5) are MMPS functions of $\tilde{u}, x, \tilde{e}$, and $\tilde{r}$.

In order to reduce the number of decision variables and the corresponding computational complexity in MPC, one often introduces a control horizon $N_{\mathrm{c}}\left(\leq N_{\mathrm{p}}\right)$ and imposes an additional condition that the input rate should be constant from step $k+N_{\mathrm{c}}-1$ on: $\Delta u(k+j)=\Delta u\left(k+N_{\mathrm{c}}-1\right)$ for $j=N_{\mathrm{c}}, \ldots, N_{\mathrm{p}}-1$. MPC uses a receding horizon principle, which means that after computation of the optimal control sequence $u(k), \ldots, u\left(k+N_{\mathrm{c}}-1\right)$, only the first control sample $u(k)$ will be implemented, subsequently the horizon is shifted one step, and the optimization is restarted after estimating or measuring the new state vector. Therefore, using a combination of the above input and output cost criteria in (5) and considering the fact that we deal with a stochastic system, we can define the stochastic MPC-MMPS problem can be defined as

$$
\begin{gathered}
\min _{\tilde{u}(k)} \tilde{J}(k) \\
\text { subject to: } c(\tilde{u}(k), k) \leq 0 .
\end{gathered}
$$

where $\tilde{J}(k)=\mathbb{E}\left[J_{\text {out }}(k)+\lambda J_{\text {in }}(k)\right]$ with $\mathbb{E}[\cdot]$ denoting the expected value operator, and $c$ is a convex function of $\tilde{u}(k)$.

\section{MMPS FUNCTIONS AND PIECEWISE AFFINE FUNCTIONS}

In this section, we show that we can write an MMPS function as a difference of two convex functions. To this end, we need the following definition and propositions.

Definition 1 (Piecewise Affine Function (PWA) [24]): A scalar-valued function $g: \mathbb{R}^{n} \rightarrow \mathbb{R}$ is said to be a 
continuous PWA function if and only if the domain space $\mathbb{R}^{n}$ is divided into a finite number of polyhedral regions $R_{1}, \ldots, R_{N}$ where for each $i \in\{1, \ldots, N\}, g$ can be expressed as $g(x)=\alpha_{i}^{T} x+\beta_{i}$ for any $x \in R_{i}$ with $\alpha_{i} \in \mathbb{R}^{n}$ and $\beta_{i} \in \mathbb{R}$ and $g$ is continuous on any boundary between two regions.

Note that for a vector-valued function $g: \mathbb{R}^{n} \rightarrow \mathbb{R}^{m}$, each component of $g$ is a continuous PWA function satisfying the above conditions.

Proposition 2 ( [25]): Any scalar-valued MMPS function can be rewritten into the min-max canonical form $g(x)=$ $\min _{i=1, \ldots, K} \max _{j \in n_{i}}\left(\alpha_{i j}^{T} x+\beta_{i j}\right)$ or into the max-min canonical form $g(x)=\max _{i=1, \ldots, L} \min _{j \in m_{i}}\left(\gamma_{i j}^{T} x+\delta_{i j}\right)$ for some integers $K, L$, non-empty subsets $n_{i}$ and $m_{i}$ of the index sets $\{1,2, \ldots, K\}$ and $\{1,2 \ldots, L\}$ respectively, real numbers $\beta_{i j}, \delta_{i j}$, and vectors $\alpha_{i j}, \gamma_{i j}$.

Proposition 3 ( [4], [5]): Any MMPS function can be written as a continuous PWA function and vice versa.

Proposition 4 ([21], [22]): The function $g(x)=$ $\min _{i=1, \ldots, K} \max _{j \in n_{i}} \delta_{i j}(x)$, where $\delta_{i j}(x)=\alpha_{i j}^{T} x+\beta_{i j}$ is an affine function in $x$, can be written as a difference of two convex functions, i.e., $g(x)=p(x)-q(x)$ where $p(x)$ and $q(x)$ are defined as follows:

$$
\begin{aligned}
p(x) & =\sum_{i=1}^{K} \max _{j \in n_{i}} \delta_{i j}(x) \\
q(x) & =p(x)-g(x) \\
& =p(x)-\min _{i=1, \ldots, K} \max _{j \in n_{i}} \delta_{i j}(x) \\
& =p(x)+\max _{i=1, \ldots, K}\left(-\max _{j \in n_{i}} \delta_{i j}(x)\right) \\
& =\max _{i=1, \ldots, K}\left(p(x)-\max _{j \in n_{i}} \delta_{i j}(x)\right) .
\end{aligned}
$$

Considering the above propositions and specifically equations (8) and (9), the following corollary is concluded:

Corollary 5: The function $g(x)$ $\max _{i=1, \ldots, L} \min _{j \in m_{i}} l_{i j}(x)$, where $l_{i j}(x)=\gamma_{i j}^{T} x+\xi_{i j}$ is an affine function in $x$, can be written as $g(x)=s(x)-r(x)$ where $s(x)$ and $r(x)$ are both convex functions defined as follows:

$$
\begin{aligned}
r(x) & =-\sum_{i=1}^{L} \min _{j \in m_{i}} l_{i j}(x) \\
& =\sum_{i=1}^{L} \max _{j \in m_{i}}\left(-l_{i j}(x)\right) \\
s(x) & =r(x)+g(x) \\
& =r(x)+\max _{i=1, \ldots, L} \min _{j \in m_{i}} l_{i j}(x) \\
& =\max _{i=1, \ldots, L}\left(r(x)+\min _{j \in m_{i}} l_{i j}(x)\right) \\
& =\max _{i=1, \ldots, L}\left(r(x)-\max _{j \in m_{i}}\left(-l_{i j}(x)\right)\right) \\
& =\max _{i=1, \ldots, L}\left(\sum_{\substack{i^{\prime}=1 \\
i^{\prime} \neq i}}^{L} \max _{j \in m_{i^{\prime}}}\left(-l_{i^{\prime} j}(x)\right)\right)
\end{aligned}
$$

$$
=\max _{k=1, \ldots, N} t_{k}(x)
$$

where $t_{k}(x)$ is an affine function in $x$. Note that the last equivalence is obtained using the distributive property of addition w.r.t. maximization.

By Proposition 2, we can write the cost criterion $\tilde{J}(k)$ in (7) as an expected value of a max-min canonical form as follows:

$$
\tilde{J}(k)=\mathbb{E}\left[\max _{i=1, \ldots, \ell} \min _{j \in m_{i}}\left(\alpha_{i j}(k)+\beta_{i j}^{T} \tilde{u}(k)+\gamma_{i j}^{T} \tilde{e}(k)\right)\right]
$$

where $\alpha_{i j}(k)$ is affine in $x(k-1)$ and $\tilde{r}(k)$. To shorten the notations, let $g(\tilde{u}(k), \tilde{e}(k))=\max _{i=1, \ldots, \ell} \min _{j \in m_{i}} \alpha_{i j}(k)+$ $\beta_{i j}^{T} \tilde{u}(k)+\gamma_{i j}^{T} \tilde{e}(k)$. Therefore, by using Corollary 5 we can rewrite (12) as:

$$
\begin{aligned}
\tilde{J}(k) & =\mathbb{E}[g(\tilde{u}(k), \tilde{e}(k))] \\
& =\mathbb{E}[s(\tilde{u}(k), \tilde{e}(k))-r(\tilde{u}(k), \tilde{e}(k))] \\
& =\mathbb{E}[s(\tilde{u}(k), \tilde{e}(k))]-\mathbb{E}[r(\tilde{u}(k), \tilde{e}(k))]
\end{aligned}
$$

where $s(\tilde{u}(k), \tilde{e}(k))$ and $r(\tilde{u}(k), \tilde{e}(k))$ are defined as given in Corollary 5 , and where the last equality comes from the fact that $\mathbb{E}[\cdot]$ is a linear operator.

Note that the cost function $\tilde{J}(k)$ in (13) results in a nonconvex optimization problem in its current structure. Now to solve the optimization problem (7) it is only left to find an efficient way to compute the expected values in (13), which will be explained in the next section. Note that $s(\tilde{u}(k), \tilde{e}(k))$ and $r(\tilde{u}(k), \tilde{e}(k))$ both consist of a maximization of affine terms. Therefore, our aim is to find an efficient way to approximate the following general expression:

$$
h=\mathbb{E}\left[\max _{j=1, \ldots, n}\left(\xi_{j}+\gamma_{j}^{T} \tilde{e}\right)\right]
$$

where $\xi_{j} \in \mathbb{R}$ and by assumption is an affine term in $\tilde{u}(k), \gamma_{j} \in \mathbb{R}^{n_{\tilde{e}}}$, and $\tilde{e}$ is a Gaussian random variable with a probability density function $f$. Note that dependence of $\xi_{j}, \gamma_{j}$, and $\tilde{e}$ on $\tilde{u}(k)$ and/or $k$ is dropped for the sake of brevity.

\section{Approximation METHOD}

Since the computation of the expected value in the cost function $\tilde{J}(k)$ in (12), and accordingly in (13) and (14), in general leads to the numerical integrations, it imposes a huge computational burden, especially when the number of stochastic variables increases. A semi-analytic approach for the integral computation is proposed in [26]. However, this method is still very time consuming and complex since at each event step many polyhedra have to be computed in order to solve the integral (see [26] for more details). Therefore, it is desired to decrease the computational burden while still having a good performance. To this end, we apply Jensen's inequality and the approximation method proposed in [23], which is based on raw moments of a random variable.

Theorem 6 (Jensen's Inequality [27]): Let $x$ be an integrable real-valued random variable and $\varphi$ a convex function such that $\varphi(x)$ is integrable. Then: $\varphi(\mathbb{E}[x]) \leq \mathbb{E}[\varphi(x)]$. Likewise, if $\varphi$ is a concave function, then $\varphi(\mathbb{E}[x]) \geq \mathbb{E}[\varphi(x)]$. 
The following theorem shows the relation between (14) and the proposed approximation method.

Theorem 7: Assume that $y=\left[y_{1}, \ldots, y_{n}\right]^{T}$ is a random vector such that all its elements are independent and identically normally distributed. Let $x_{j}=y_{j}-L$ where $L \in \mathbb{R}$. The following inequalities hold:

$$
\begin{aligned}
\max \left(\mathbb{E}\left[x_{1}\right], \ldots, \mathbb{E}\left[x_{n}\right]\right) & \stackrel{(i)}{\leq} \mathbb{E}\left[\max \left(x_{1}, \ldots, x_{n}\right)\right] \\
& \stackrel{(i i)}{\leq} \mathbb{E}\left[\max \left(\left|x_{1}\right|, \ldots,\left|x_{n}\right|\right)\right] \\
& \stackrel{(\text { iii })}{\leq} \mathbb{E}\left[\left(\left|x_{1}\right|^{p}+\cdots+\left|x_{n}\right|^{p}\right)^{1 / p}\right] \\
& \stackrel{(\text { iv })}{\leq}\left(\sum_{j=1}^{n} \mathbb{E}\left[\left|x_{j}\right|^{p}\right]\right)^{1 / p}
\end{aligned}
$$

Proof: Inequalities $(i)$ and (iv) result from Jensen's inequality for convex and concave functions, respectively. Inequality $($ iii) results from the relation between $\infty$-norm and $p$-norm for $p \geq 1$ [28], i.e., $\|x\|_{\infty} \leq\|x\|_{p} \leq n^{1 / p}\|x\|_{\infty}$.

Remark 8: In case that $y_{j}, j=1, \ldots, n$ is a bounded random variable, the inequality (ii) turns into an equality. However, this is not the case since we are considering Gaussian noise. Hence, in order to decrease the error of approximating $y$ by $|y|$, we introduce a scalar $L$. Since in our case, it is assumed that $y_{j} \sim \mathscr{N}\left(\mu_{j}, \sigma_{j}^{2}\right)$ for $j=1, \ldots, n$, we define $L=\min _{j=1, \ldots, n}\left(\mu_{j}-3 \sigma_{j}\right)$. This choice has been made due to $3 \sigma$-rule which indicates that $99.7 \%$ of observations of a normally distributed random variable fall within the mean minus 3 times the standard deviation and the mean plus 3 times the standard deviation.

Hence, we can approximate (14) by computing its lower and upper bound, using the left-hand side and the right-hand side of (15), as follows:

$$
\begin{aligned}
h_{\mathrm{low}} & =\max _{j=1, \ldots, n}\left(\mathbb{E}\left[\xi_{j}+\gamma_{j}^{T} \tilde{e}\right]\right) \\
h_{\mathrm{up}} & =\left(\sum_{j=1}^{n} \mathbb{E}\left[\left(\xi_{j}+\gamma_{j}^{T} \tilde{e}-L\right)^{p}\right]\right)^{1 / p}
\end{aligned}
$$

which are both convex in $\tilde{u}$ [23]. Since for an even positive integer $p, \mathbb{E}\left[x^{p}\right]=\mathbb{E}\left[|x|^{p}\right]$, in the rest of the paper we consider $\mathbb{E}\left[x^{p}\right]$ and we assume that $p$ is an even integer larger than or equal to 2 .

As mentioned before, we assume that the elements of the stochastic vector $e$ are independent and identically normally distributed, i.e., $e_{\ell} \sim \mathscr{N}\left(\mu_{\ell}, \sigma_{\ell}^{2}\right)$ for $\ell=1, \ldots, n_{e}$. Accordingly, the computation of (16) is straightforward, and we only need to elaborate on the computation of (17). By using the property of the normal distribution that sum of the independent normally distributed random variables also has a normal distribution [29], we conclude that the random variable $\omega_{j}=$ $\xi_{j}+\gamma_{j}^{T} \tilde{e}-L$ in (16) is also normally distributed with mean $\mu_{j}=\xi_{j}+\gamma_{j}^{T} \mu_{e}-L$ and variance $\sigma_{j}^{2}=\sum_{\ell=1}^{n_{e}}\left(\gamma_{j}\right)_{\ell}^{2} \sigma_{\ell}^{2}$, where $\mu_{e}=\left[\mu_{1}, \ldots, \mu_{n_{e}}\right]^{T}$ and $L=\min _{j=1, \ldots, n}\left(\xi_{j}+\gamma_{j}^{T} \mu_{e}-3 \sigma_{j}\right)$. Note that the expected value $\mathbb{E}\left[\omega_{j}\right]$ in (16) is then nothing but the $p$-th raw moment of a normally distributed random variable, which is by definition equal to

$$
\mathbb{E}\left[x^{p}\right]=\int_{-\infty}^{\infty} x^{p} \frac{1}{\sqrt{2 \pi} \sigma} e^{-(x-\mu)^{2} /\left(2 \sigma^{2}\right)} \mathrm{d} x
$$

for $x \sim \mathscr{N}\left(\mu, \sigma^{2}\right)$ and finite for all even integers $p$. Since we intend to improve the time efficiency and to decrease the computational complexity by avoiding numerical integration, we use the closed form of the above moment, given by [30]:

$$
\mathbb{E}\left[x^{p}\right]=\sigma^{p} i^{-p} H_{p}(i \mu / \sigma)
$$

where

$$
\begin{aligned}
H_{p}(x) & \equiv(-1)^{p} \exp \left(x^{2} / 2\right) \frac{d^{p}}{d x^{p}} \exp \left(-x^{2} / 2\right) \\
& =p ! \sum_{k=0}^{p / 2} \frac{(-1)^{k} x^{p-2 k}}{2^{k} k !(p-2 k) !}
\end{aligned}
$$

is the $p$-th order Hermite polynomial, and where the second equality can be obtained by considering equations (26.2.51) and (22.3.11) in [31]. Note that the right-hand side of (14) is real-valued because $H_{p}(x)$ contains only even powers of $x$ if $p$ is even. Accordingly, we can rewrite (17) as

$$
h_{\text {up }}=\left(\sum_{j=1}^{n} \sigma_{j}^{p} i^{-p} H_{p}\left(i \mu_{j} / \sigma_{j}\right)\right)^{1 / p}
$$

In an example we illustrate the procedure given above.

Example: Let $g(\tilde{u}, \tilde{e})=\max \left(\min \left(\gamma_{1} \tilde{e}+\xi_{1}, \gamma_{2} \tilde{e}+\right.\right.$ $\left.\left.\xi_{2}\right), \min \left(\gamma_{3} \tilde{e}+\xi_{3}, \gamma_{4} \tilde{e}+\xi_{4}\right)\right)$, where $\tilde{e}$ is a stochastic vector and all its elements are i.i.d normally distributed with mean $\mu_{\ell}$ and variance $\sigma_{\ell}^{2}, \ell=1, \ldots, n_{\tilde{e}}, \xi_{j} \in \mathbb{R}$, and $\gamma_{j} \in \mathbb{R}^{n_{\tilde{e}}}$ for $j=1, \ldots, 4$. Following Corollary (5), we can rewrite $g(\tilde{u}, \tilde{e})$ as $g(\tilde{e})=s(\tilde{u}, \tilde{e})-r(\tilde{u}, \tilde{e})$ where $s$ and $r$ are convex functions defined as follows:

$$
\begin{aligned}
r(\tilde{u}, \tilde{e})= & \max \left(-\gamma_{1}^{T} \tilde{e}-\xi_{1},-\gamma_{2}^{T} \tilde{e}-\xi_{2}\right) \\
& +\max \left(-\gamma_{3}^{T} \tilde{e}-\xi_{3},-\gamma_{4}^{T} \tilde{e}-\xi_{4}\right) \\
s(\tilde{u}, \tilde{e})= & r(\tilde{u}, \tilde{e})+g(\tilde{u}, \tilde{e}) \\
= & \max \left(\max \left(-\gamma_{1}^{T} \tilde{e}-\xi_{1},-\gamma_{2}^{T} \tilde{e}-\xi_{2}\right),\right. \\
& \left.\quad \max \left(-\gamma_{3}^{T} \tilde{e}-\xi_{3},-\gamma_{4}^{T} \tilde{e}-\xi_{4}\right)\right) \\
= & \max \left(-\gamma_{1}^{T} \tilde{e}-\xi_{1},-\gamma_{2}^{T} \tilde{e}-\xi_{2},-\gamma_{3}^{T} \tilde{e}-\xi_{3},\right. \\
& \left.\left.\quad-\gamma_{4}^{T} \tilde{e}-\xi_{4}\right)\right)
\end{aligned}
$$

Hence an upper bound for $\mathbb{E}[g(\tilde{u}, \tilde{e})]$ can be obtained by computing an upper bound for $\mathbb{E}[s(\tilde{u}, \tilde{e})]$ and a lower bound for $\mathbb{E}[r(\tilde{u}, \tilde{e})]$ where

$$
\begin{aligned}
\{\mathbb{E}[s(\tilde{u}, \tilde{e})]\}_{\text {up }}= & \left(\sum_{j=1}^{4} \mathbb{E}\left[\left(-\gamma_{j}^{T} \tilde{e}-\xi_{j}\right)^{p}\right]\right)^{1 / p} \\
\{\mathbb{E}[r(\tilde{u}, \tilde{e})]\}_{\text {low }}= & \max \left(-\gamma_{1}^{T} \mathbb{E}[\tilde{e}]-\xi_{1},-\gamma_{2}^{T} \mathbb{E}[\tilde{e}]-\xi_{2}\right) \\
& +\max \left(-\gamma_{3}^{T} \mathbb{E}[\tilde{e}]-\xi_{3},-\gamma_{4}^{T} \mathbb{E}[\tilde{e}]-\xi_{4}\right)
\end{aligned}
$$

where $\mathbb{E}[\tilde{e}]=\left[\mu_{1}, \ldots, \mu_{n_{e}}\right]^{T}$.

Consequently, instead of minimizing the cost function $\tilde{J}(k)$, we will minimize its upper bound $\tilde{J}_{\text {up }}(k)$, which, considering (13), can be obtained by computing an upper bound for $\mathbb{E}[s(\tilde{u}(k), \tilde{e}(k))]$, given in (19), and a lower bound for 
$\mathbb{E}[r(\tilde{u}(k), \tilde{e}(k))]$, given in (16). In this way the optimization problem turns into a sequence of convex problems as follows:

$$
\begin{aligned}
& \min _{\tilde{u}(k)} \tilde{J}_{\text {up }}(k) \\
& =\min _{\tilde{u}(k)}\left(\{\mathbb{E}[s(\tilde{u}(k), \tilde{e}(k))]\}_{\text {up }}-\{\mathbb{E}[r(\tilde{u}(k), \tilde{e}(k))]\}_{\text {low }}\right) \\
& =\min _{\tilde{u}(k)}\left(h_{\text {up }}(\tilde{u}(k))-\sum_{i=1}^{M} \max _{k \in n_{i}}\left(\mathbb{E}\left[t_{k}(\tilde{u}(k), \tilde{e}(k))\right]\right)\right) \\
& =\min _{\tilde{u}(k)}\left(h_{\text {up }}(\tilde{u}(k))+\sum_{i=1}^{M} \min _{k \in n_{i}}\left(-\mathbb{E}\left[t_{k}(\tilde{u}(k), \tilde{e}(k))\right]\right)\right) \\
& =\min _{\tilde{u}(k)}\left(h_{\text {up }}(\tilde{u}(k))+\min _{j=1, \ldots, N}\left(-\mathbb{E}\left[v_{j}(\tilde{u}(k), \tilde{e}(k))\right]\right)\right) \\
& =\min _{\tilde{u}(k)} \min _{j=1, \ldots, N}\left(h_{\text {up }}(\tilde{u}(k))-\mathbb{E}\left[v_{j}(\tilde{u}(k), \tilde{e}(k))\right]\right) \\
& =\min _{j=1, \ldots, N} \min _{\tilde{u}(k)}\left(h_{\text {up }}(\tilde{u}(k))-\mathbb{E}\left[v_{j}(\tilde{u}(k), \tilde{e}(k))\right]\right)
\end{aligned}
$$

where $t_{k}(\tilde{u}(k), \tilde{e}(k))$ and $v_{j}(\tilde{u}(k), \tilde{e}(k))$ are affine functions. Note that $\min _{\tilde{u}(k)}\left(h_{\text {up }}(\tilde{u}(k))-\mathbb{E}\left[v_{j}(\tilde{u}(k), \tilde{e}(k))\right]\right)$ is a convex optimization problem. Hence by adding the convex constraints, mentioned in (7), to the optimization problem (20), we can solve it efficiently for each $j=1, \ldots, N$ using convex optimization algorithms such as interior point methods [32].

\section{EXAMPLE: STOCHASTIC MPC-MMPS PROBLEM}

In this example we study the control of the temperature of a room (see [3]). There, the following continuous discretetime PWA system is obtained:

$$
\begin{aligned}
x(k+1) & = \begin{cases}1 / 2 x(k)+u(k)+e_{1}(k)+1 & \text { if } x(k)<0 \\
u(k)+e_{1}(k)+1 & \text { if } x(k) \geq 0\end{cases} \\
y(k) & =x(k)+e_{2}(k) .
\end{aligned}
$$

with the following constraints on the input:

$-4 \leq \Delta u(k)=u(k+1)-u(k) \leq 4$ and $u(k) \geq 0$ for all $k$.

Note that this example is similar to the one in [3], except that here we assume that the error components have standard normal distribution, i.e., $e_{1}(k), e_{2}(k) \sim \mathscr{N}(0,1)$. So for the intermediate steps to obtain (21)-(22), the interested reader is referred to [3]. The equivalent MMPS representation of (21)-(22) is the following:

$$
\begin{aligned}
x(k+1) & =\min \left(1 / 2 x(k)+u(k)+e_{1}(k)+1, u(k)+e_{1}(k)+1\right), \\
y(k) & =x(k)+e_{2}(k) .
\end{aligned}
$$

Since at sample step $k$ the input $u(k)$ has no influence on $y(k)$, we choose $N_{\mathrm{p}}=3, N_{\mathrm{c}}=2, \tilde{y}(k)=[\hat{y}(k+1) \hat{y}(k+2)]^{T}$, $\tilde{r}(k)=[r(k+1) r(k+2)]^{T}, \tilde{u}(k)=[u(k) u(k+1)]^{T}$. Let the uncertainty vector $e(k)$ be $e(k)=\left[e_{1}(k) e_{2}(k+1)\right]^{T}$. Therefore, $\tilde{e}(k)=\left[e^{T}(k) e^{T}(k+1)\right]^{T}$. As a cost criterion we make a similar choice as [3], i.e.,

$$
\tilde{J}(k)=\mathbb{E}\left[\|\tilde{y}(k)-\tilde{r}(k)\|_{\infty}+\lambda\|\tilde{u}(k)\|_{1}\right] .
$$

where in the cost criterion we have the expected value due to the stochastic setting while in [3] instead of expected value, the worst-case value over all disturbances is used due to the different uncertainty setting of [3]. Since $u(k) \geq 0$, we have
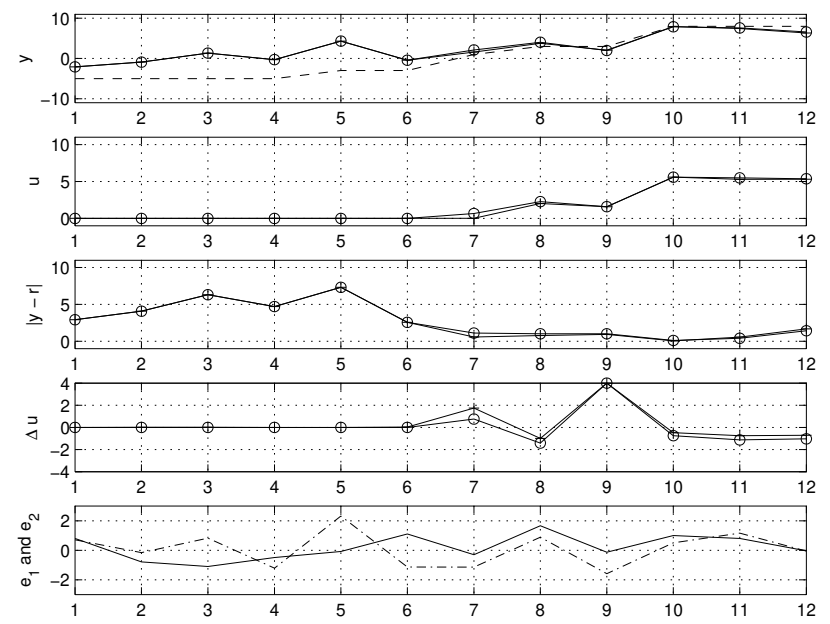

Fig. 1. Closed-loop optimization results of the stochastic MPC-MMPS problem. 'o'-line: upper bound approach; '+'-line: exact solution using numerical integration; dashed line: reference signal; dash-dot and solid line: error components

$\|u(k)\|_{1}=u(k)$ and therefore we get the following max-min expression for $\tilde{J}(k)$ :

$$
\begin{array}{r}
\tilde{J}(k)=\mathbb{E}[\max (|\tilde{y}(k)-\tilde{r}(k)|)+\lambda(u(k)+u(k+1))] \\
=\mathbb{E}[\max (y(k+1)-r(k+1)+\lambda u(k)+\lambda u(k+1), \\
y(k+2)-r(k+2)+\lambda u(k)+\lambda u(k+1), \\
-y(k+1)+r(k+1)+\lambda u(k)+\lambda u(k+1), \\
-y(k+2)+r(k+2)+\lambda u(k)+\lambda u(k+1))]
\end{array}
$$

We compute the closed-loop MPC controller by minimizing the upper bound of the criterion function (as shown in (20)) over a simulation period $[1,20]$, with $\lambda=0.01$, $x(0)=-6, u(-1)=0$ and the reference signal $\{r(k)\}_{k=1}^{20}=$ $\{-5,-5,-5,-5,-3,-3,1,3,3,8,8,8,8,10,10,7,7,7,4,3\}$.

Figure 1 shows the results of the closed-loop simulation in which the exact optimal solution obtained from numerical integration is compared to the one obtained from the upper bound approach in (20). The top plot shows the reference signal (dashed line), the output of the system using numerical integration ('+'-line), and the one using the upper bound approach ('o'-line). The next two plots present the optimal input sequence and tracking error, respectively, using numerical integration ('+'-line) and the upper bound approach ('o'-line). The last two plots show $\Delta u^{*}(k)=u^{*}(k+1)-u^{*}(k)$ and the error vector, respectively. The computation time ${ }^{1}$ using the numerical integration (i.e., the exact solution) is $23447 s$ compared to $323.02 s$ using the upper bound approach. Moreover, based on the above plots, the results of the upper bound approach in (20) is very close to the one from numerical integration. Hence, we can solve the stochastic MPC-MMPS problem using (20) in order to decrease the computation time while still guaranteeing a good performance.

\footnotetext{
${ }^{1}$ These times were obtained running Matlab 7.5.0 (R2007b) on a 2.33 GHz Intel Core Duo E655 processor.
} 


\section{CONCLUSIONS}

We have proposed an approximation method to solve the stochastic MPC-MMPS optimization problem in particular. Accordingly, computation of the cost criterion, which is defined as an expected value of an MMPS function, involves numerical integration that is both complex and time consuming. By rewriting an MMPS function as a difference of two convex functions, the problem has been narrowed down to finding the expected value of these two functions. As a solution we proposed to optimize the upper bound of the criterion function instead of the criterion function itself, which leads to computing the upper bound of one of the convex functions by applying an approximation method based on raw moments of a random variable, and the lower bound of the other convex function. These bounds resulted in an analytic expressions and consequently, the computational complexity and the computation time have been decreased considerably while we still have a good performance. In our future research we will apply this approach to a higher dimensional and more complex example.

\section{ACKNOWLEDGMENTS}

This research is partially funded by the Dutch Technology Foundation STW project "Model-predictive railway traffic management" (11025), and by the European 7th Framework Network of Excellence project "Highly-complex and networked systems (HYCON2)".

\section{REFERENCES}

[1] J. M. Maciejowski, Predictive Control with Constraints. Harlow, England: Prentice Hall, 2002.

[2] B. De Schutter and T. van den Boom, "Model predictive control for max-plus-linear discrete event systems," Automatica, vol. 37, no. 7, pp. 1049-1056, Jul. 2001.

[3] I. Necoara, B. De Schutter, T. van den Boom, and H. Hellendoorn, "Model predictive control for uncertain max-min-plus-scaling systems," International Journal of Control, vol. 81, no. 5, pp. 701-713, May 2008.

[4] V. Gorokhovik and O. Zorko, "Piecewise affine functions and polyhedral sets," Optimization, vol. 31, pp. 209-221, 1994.

[5] S. Ovchinnikov, "Max-min representation of piecewise linear functions," Beiträge zur Algebra und Geometrie/Contributions to Algebra and Geometry, vol. 43, no. 1, pp. 297-302, 2002.

[6] W. Heemels, B. De Schutter, and A. Bemporad, "Equivalence of hybrid dynamical models," Automatica, vol. 37, no. 7, pp. 1085-1091, Jul. 2001.

[7] A. Bemporad and M. Morari, "Control of systems integrating logic, dynamics, and constraints," Automatica, vol. 35, no. 3, pp. 407-427, Mar. 1999.

[8] M. Johansson, Piecewise Linear Control Systems, ser. Lecture Notes in Control and Information Sciences. Berlin, Germany: Springer, 2003, vol. 284.

[9] G. J. Olsder, J. A. C. Resing, R. E. de Vries, M. Keane, and G. Hooghiemstra, "Discrete event systems with stochastic processing times," IEEE Transactions on Automatic Control, vol. 35, no. 3, pp. 299-302, Mar. 1990.

[10] J. A. C. Resing, R. E. de Vries, G. Hooghiemstra, M. S. Keane, and G. J. Olsder, "Asymptotic behavior of random discrete event systems," Stochastic Processes and Their Applications, vol. 36, no. 2, pp. 195216, Dec. 1990

[11] F. Baccelli, G. Cohen, G. Olsder, and J. Quadrat, Synchronization and Linearity. New York: John Wiley \& Sons, 1992.

[12] B. Heidergott, G. J. Olsder, and J. van der Woude, Max Plus at Work. Princeton, New Jersey: Princeton University Press, 2006.
[13] J. Mairesse, "Stochastic linear systems in the (max,+) algebra," in 11th International Conference on Analysis and Optimization of Systems Discrete Event Systems, ser. Lecture Notes in Control and Information Sciences. Springer-Verlag, 1994, vol. 199, pp. 257-265.

[14] E. Kerrigan and D. Mayne, "Optimal control of constrained, piecewise affine systems with bounded disturbances," in Proceedings of the 41st IEEE Conference on Decision and Control, Las Vegas, Nevada, Dec. 2002, pp. 1552-1557.

[15] S. V. Rakovic, E. C. Kerrigan, and D. Q. Mayne, "Optimal control of constrained piecewise affine systems with state- and input-dependent disturbances," in Proceedings of the 16th International Symposium on Mathematical Theory of Networks and Systems, Leuven, Belgium, Jul. 2004.

[16] I. Necoara, E. Kerrigan, B. De Schutter, and T. van den Boom, "Finite-horizon min-max control of max-plus-linear systems," IEEE Transactions on Automatic Control, vol. 52, no. 6, pp. 1088-1093, Jun. 2007.

[17] S. Young and V. Garg, "Model uncertainty in discrete event systems," SIAM Journal on Control and Optimization, vol. 33, no. 1, pp. 208226, Jan. 1995.

[18] A. Bemporad and S. D. Cairano, "Model-predictive control of discrete hybrid stochastic automata," IEEE Trans. Automatic Control, vol. 56 no. 6, pp. 1307-1321, 2011.

[19] B. Kouvaritakis, M. Cannon, and V. Tsachouridis, "Recent developments in stochastic mpc and sustainable development," Annual Reviews in Control, vol. 28, no. 1, pp. 23-35, 2004.

[20] B. De Schutter and T. van den Boom, "Model predictive control for max-min-plus-scaling systems - Efficient implementation," in Proceedings of the 6th International Workshop on Discrete Event Systems (WODES'02), M. Silva, A. Giua, and J. Colom, Eds., Zaragoza, Spain, Oct. 2002, pp. 343-348.

[21] A. Kripfganz and R. Schulze, "Piecewise affine functions as a difference of two convex functions," Optimization, vol. 18, no. 1, pp. 23-29, 1987.

[22] C. Wen, S. Wang, F. Li, and M. Khan, "A compact f-f model of highdimensional piecewise-linear function over a degenerate intersection," IEEE Transactions on Circuits and Systems-I: Fundamental Theory and Applications, vol. 52, no. 4, pp. 815-821, Apr. 2005.

[23] S. S. Farahani, T. van den Boom, H. van der Weide, and B. De Schutter, "An approximation approach for model predictive control of stochastic max-plus linear systems," in Proceedings of the workshop on Discrete Event Systems (WODES), Berlin, Germany, 2010, pp. 386391

[24] L. Chua and A. Deng, "Canonical piecewise-linear representation," IEEE Transactions on Circuits and Systems, vol. 35, no. 1, pp. 101111, Jan. 1988.

[25] B. De Schutter and T. van den Boom, "MPC for continuous piecewiseaffine systems," Systems \& Control Letters, vol. 52, no. 3-4, pp. 179192, Jul. 2004.

[26] T. J. J. van den Boom and B. De Schutter, "Model predictive control for perturbed max-plus-linear systems: A stochastic approach," International Journal of Control, vol. 77, no. 3, pp. 302-309, Feb. 2004.

[27] S. Boyd and L. Vandenberghe, Convex Optimization. London, UK: Cambridge University Press, 2004.

[28] G. H. Golub and C. F. Van Loan, Matrix Computations. Baltimore, Maryland: The John Hopkins University Press, 1990.

[29] F. Dekking, C. Kraaikamp, H. P. Lopuhaä, and L. E. Meester, A Modern Introduction to Probability and Statistics. London: Springer, 2005 .

[30] R. Willink, "Normal moments and Hermite polynomials," Statistics \& Probability Letters, vol. 73, no. 3, pp. 271-275, Jul. 2005.

[31] M. A. Abramowitz and I. Stegun, Handbook of Mathematical Functions. Washington DC: National Bureau of Standards, US Government Printing Office, 1964.

[32] Y. Nesterov and A. Nemirovskii, Interior-Point Polynomial Algorithms in Convex Programming. Philadelphia, Pennsylvania: SIAM, 1994. 